\title{
Recovery of Frontal Cortex-mediated Visual Behaviors following Neurotrophic Rescue of Axotomized Neurons in Medial Frontal Cortex
}

\author{
Forrest Haun and Timothy J. Cunningham \\ Department of Anatomy and Neurobiology, Medical College of Pennsylvania, Philadelphia, Pennsylvania 19129
}

\begin{abstract}
Unilateral lesions extending across the boundary region of visual and parietal cortex in adult rats result in the death of $20-35 \%$ of neurons in layers II-III of the caudal third of medial frontal cortex ipsilaterally, a neuron population labeled with ${ }^{3} \mathrm{H}$-thymidine on the 19th day of gestation (E19). Additionally, there is a consistent $15 \%$ loss of these labeled neurons in an area between $50 \%$ and $60 \%$ of the distance along the caudal-rostral extent of medial frontal cortex, an area that may function analogously to the frontal eye field of primates. All of these neurons are rescued from axotomyinduced death by delivering into the posterior cortex lesion cavity for 2 weeks a macromolecular fraction of culture medium conditioned by embryonic primordia of the frontal-occipital pathway (CM). Moreover, the rescue is apparently permanent, with normal numbers of these neurons present in CM animals 6-7 weeks after the neurotrophic factor is no longer being supplied exogenously. Behaviorally, control operates receiving a similarly prepared fraction of unconditioned medium are significantly impaired in the number of trials needed to learn two visual discrimination tasks. This deficit is attributable in part to a bias in erroneous responses to the side contralateral to the lesion. The error bias reflects a failure to inhibit repeated incorrect responding contralaterally. In contrast, the $\mathrm{CM}$ animals learn both visual tasks in a normal number of trials and have no contralateral error bias. Rather, all CM animals have an ipsilateral error bias (interpreted as an unmasking of the contralateral neglect expected after a parietal cortex lesion). These results demonstrate that (1) adult cortical neurons are rescued chronically from axotomy-induced death by an acutely provided diffusible factor derived from embryonic cortex, and (2) this factor also produces recovery of behaviors characteristic of the pathway from which the neurotrophic factor was derived.

[Key words: medial frontal cortex, visual cortex, posterior parietal cortex, neurotrophic factor, visual discrimination, contralateral neglect, functional recovery]
\end{abstract}

\footnotetext{
Received Mar. 16, 1992; revised July 29, 1992; Accepted Aug. 4, 1992.

This work was supported by NIMH Grant MH 44734 (F.H.) and NIH Grant NS 16487 (T.J.C.). We are particularly grateful to Amy Wolfram for conducting the lengthy pilot experiments for this study, Rachel Perry and Terry Jaret for dedicated assistance in running the behavioral assay, and Sarah Goldsmith and Jennifer Nuss for histological assistance. Dr. Phyllis Salzman has also provided continuing technical support.

Correspondence should be addressed to Forrest Haun, Ph.D., Department of Anatomy and Neurobiology, The Medical College of Pennsylvania, 3200 Henry Avenue, Philadelphia, PA 19129.

Copyright (C) 1993 Society for Neuroscience $0270-6474 / 93 / 130614-09 \$ 05.00 / 0$
}

There has been increasing interest in recent years in the possibility of neurotrophic intervention in the adult brain after damage, as a means of enhancing functional recovery (Hefti et al., 1989; Thoenen, 1991). In order to understand the potential of such a strategy, as well as the role(s) of neurotrophic agents in normal mature systems, it is important to have some idea of the long-term consequences of neurotrophic dependencies in adults after lesions. One plausible role for these factors is to maintain a balance of appropriate afferent and efferent contact so that a neuron's activity may contribute to advantageous behaviors. This might imply, for example, that exogenous application of a neurotrophic factor following a lesion could induce functional reorganization by axotomized neurons, such that an appropriate behavioral consequence would result. In such a scheme, the first requirement is that the damaged neurons remain alive long enough to reorganize, and then stay alive in order to support behavioral recovery over the long term.

Mature neurons may in fact die following axotomy, both in the PNS (Purves, 1975; Aldskogius and Arvidsson, 1978; Himes and Tcssler, 1989) and in the CNS (Lashlcy, 1941; Blcicr, 1969; Himes et al., 1990; Eagleson et al., 1992). It has been suggested that one reason axotomy may result in death is that the injury separates the neuron from its source of (target-derived) trophic support (Cunningham et al., 1988; Purves, 1988; Varon et al., 1989). The idea that even mature neurons may normally depend on target-derived trophic factors for survival and/or mature functioning has only recently acquired empirical support. In the CNS, for example, delivery of NGF, the best characterized neurotrophic factor, into fimbria-fornix-lesioned adult rats increases neurotransmitter-related enzyme activity in septal cholinergic neurons (Hefti, 1986; Kromer, 1987; Rosenberg et al., 1988) and reduces the extent of lesion-induced atrophy in both cholinergic and noncholinergic septal neurons (Gage et al., 1988). In the geniculocortical pathway of adult rats, infusing a cortexderived neuron survival factor into a visual cortex lesion site prevents the death of axotomized neurons in the dLGN (Eagleson et al., 1992).

There is conflicting evidence on whether there may be longterm consequences on neuron survival and function following short-term application of trophic factors. An earlier report of a persisting elevation in neurotransmitter enzyme levels in septal cholinergic neurons after a 2 week delivery of NGF into fimbriafornix-lesioned rats (Will and Hefti, 1985) was not confirmed in a subsequent study (Montero and Hefti, 1988). However Eagleson et al. (1992) found that a 2 week delivery of a neuron survival factor leads to increased survival by axotomized dLGN neurons up to 6 weeks after infusion has ceased. 
In the study reported here, we examined both long-term neuron survival and chronic behavior effects in adult rats that received a 2 week infusion of a trophically active fraction of conditioned medium, directed into a posterior cortex lesion site. Postlesion behavioral testing occurred over a 2-3 month period, after which ${ }^{3} \mathrm{H}$-thymidine-labeled neurons were counted in the medial frontal cortex, a region that contains neurons that project to the lesion site in the posterior cortex.

Preliminary data from this study have been reported in abstract form (Haun et al., 1989b).

\section{Materials and Methods}

Subjects. Twenty-one adult Long-Evans rats provided data for this study. Six were normal controls, eight received lesions along the boundary between parietal and occipital cortex in the right hemisphere followed by osmotic pump implants containing cortex-conditioned medium (CM), and seven received similar lesions and pump implants containing unconditioned culture medium (UM). The operated animals had been exposed to ${ }^{3} \mathrm{H}$-thymidine in utero, in order to label neurons in particular layers of cortex as the cells underwent their final mitosis (Bayer and Altman, 1991).

Surgery and ${ }^{3} \mathrm{H}$-thymidine labeling. For thymidine labeling, two pregnant dams at $19 \mathrm{~d}$ gestation (E19, with the first day postconception considered E1) were anesthetized with chloral hydrate $(35 \mathrm{mg} / 100 \mathrm{gm}$ body weight) and then injected intraperitoneally with ${ }^{3} \mathrm{H}$-thymidine ( 1 $\mathrm{mCi} / 100 \mathrm{gm}$ body weight; specific activity, $65-80 \mathrm{Ci} / \mathrm{mmol})$. A pilot study had shown that injection at E19 results in heavy neuronal labeling in layers II-III of medial frontal cortex, especially caudally.

When the animals were at least $120 \mathrm{~d}$ old, they received lesions across the boundary region between parietal and occipital cortex in the right hemisphere, to axotomize frontal cortex neurons that project to the lesion site and to visual cortex areas caudal to the lesion. [Injections of retrograde tracers into these areas have been shown to label projection neurons in medial frontal cortex (Miller and Vogt, 1984; Dreher et al., 1990).] The animals were first anesthetized using chloral hydrate, as above, and placed in a stereotaxic apparatus. The skin overlying the skull was reflected and a medial-lateral strip drilled across the skull over the right posterior cortex at $4.2 \mathrm{~mm}$ posterior to bregma. Using a metal cannula, a suction lesion was then made in the cortex, extending from $1.2 \mathrm{~mm}$ to $4.8 \mathrm{~mm}$ lateral of the midline. The width of the lesion was the width of the suction cannula $(2 \mathrm{~mm})$, and all lesions extended down to the visible white matter. Reconstructions indicated this procedure yielded comparable lesion sizes and extent (Fig. 1; see Results).

The lesion cavity was packed with Gelfoam soaked in unconditioned culture medium (see below), and then an osmotic minipump (Alzet \#2002) was implanted in the fascia of the neck muscles, with attached tubing and cannula directed into the medial portion of the lesion cavity (see Eagleson et al., 1992, for detailed procedures). The average expected delivery time for the contents of the pump was $19.5 \mathrm{~d}$, based on the nominal pumping rates and reservoir volumes for the particular minipumps used.

The overlying skin was then sutured and the animal was placed on a heating pad for $1-2 \mathrm{hr}$ and then returned to its home cage after receiving an injection of ampicillin ( $2 \mathrm{cc}$, i.m.).

Conditioned culture medium. A plausible source of neurotrophic activity in a CNS pathway is embryonic tissue primordia from which that pathway will normally develop (Cunningham et al., 1987, 1988). For the frontal-occipital cortex pathway, then, embryonic frontal and posterior cortex pieces were dissected from E14 Long-Evans rat embryos and cocultured for $5 \mathrm{~d}$, and the conditioned culture medium was collected and a macromolecular fraction loaded into the osmotic minipumps. The embryos were removed from dams anesthetized as above, and the medial third of each frontal cortex (rostral to the middle cerebral artery) and the caudal half of each posterior cortex dissected. After meninges and blood vessels were removed, pairs of posterior and frontal pieces (approximately $1 \mathrm{~mm} \times 0.5 \mathrm{~mm} \times 0.5 \mathrm{~mm}$ each) were cocultured as explants, 3-5 mm apart, one pair per $35 \mathrm{~mm}$ Primaria dish (Falcon). Culture medium was $1.2 \mathrm{ml}$ of $90 \%$ Ham's F-10 and $10 \%$ Nu-Serum (Collaborative Research), supplemented as described in Eagleson et al. (1990). After $5 \mathrm{~d}$ in vitro in a $90 \%$ air, $10 \% \mathrm{CO}_{2}$ high-humidity atmosphere, the conditioned culture medium (CM) was collected in sterile tubes, filtered through a $0.22 \mu \mathrm{m}$ Millex-GV filter (Millipore), and then

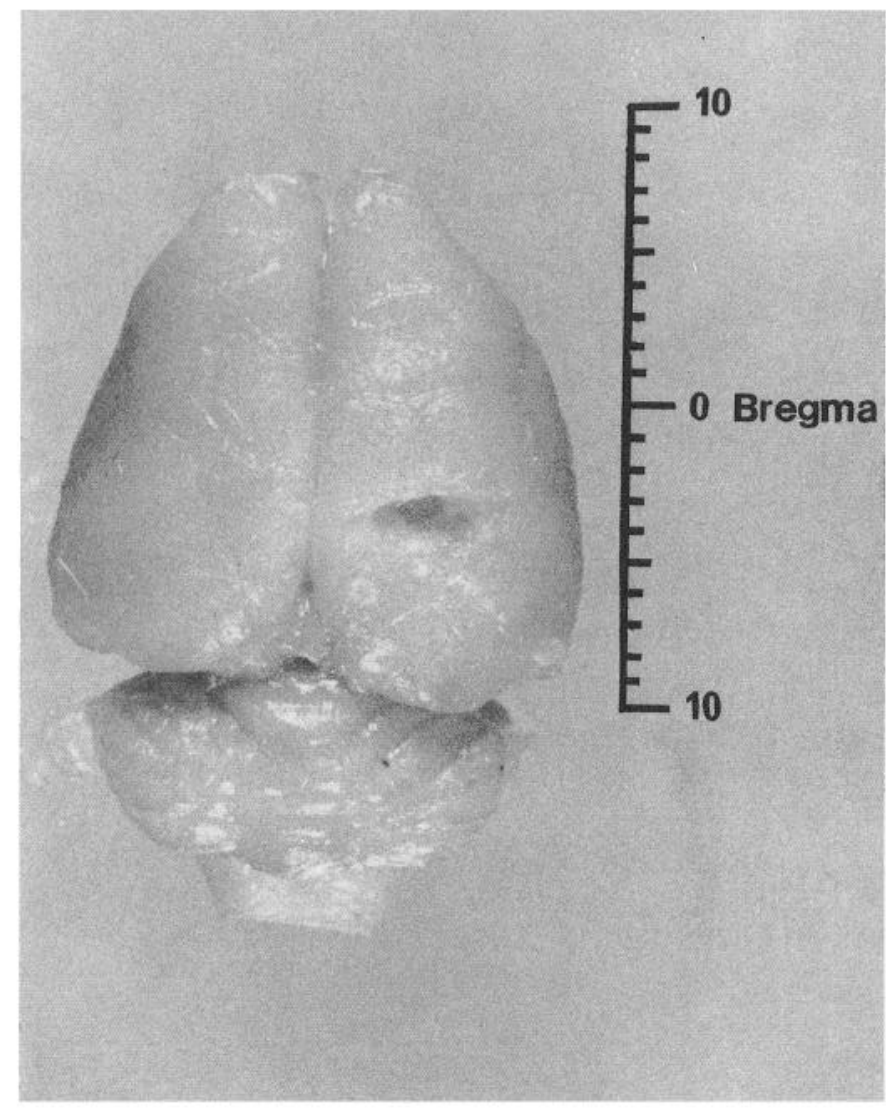

Figure 1. Location of rostral visual/posterior parietal cortex lesion, intended to disconnect frontal and occipital areas. Standard stereotaxic coordinates are used for all animals (see Materials and Methods). This animal received a UM fraction infusion and was impaired on both visual discrimination tasks.

concentrated $10 \times$ by pressure through an ultrafiltration membrane with a $10 \mathrm{kDa}$ cutoff(Pharmacia). The macromolecular fraction retained was stored as frozen droplets in liquid nitrogen. For the seven operated animals receiving this $\mathrm{CM}$ in this study, the $\mathrm{CM}$ was derived from cultures established from four separate litters of E14 embryos.

A macromolecular fraction of unconditioned culture medium (UM) was similarly prepared from culture medium that had also been placed in tissue culture dishes in an incubator for $5 \mathrm{~d}$.

Behavior testing: apparatus. Behavioral effects of the CM factor were assessed using visual discrimination tasks in which two stimuli were simultaneously presented ipsilateral and contralateral to the lesion. Performance was tested in a fully automated apparatus (Fig. 2) using a 26 cm (length) $\times 25 \mathrm{~cm}$ (width) $\times 19 \mathrm{~cm}$ (height) metal box with a grid floor (Lafayette). At one end of the box were two projection panels, each $10 \mathrm{~cm}$ (height) $\times 7.2 \mathrm{~cm}$ (width) separated by a $1.3 \mathrm{~cm}$ divider. Centered directly underneath the divider was a $4.8 \mathrm{~cm}$ metal lever that, when pressed, initiated presentation of two visual stimuli, one on each projection panel. The stimuli were back-projected from a Kodak Carousel such that each stimulus filled the entire panel. On each side wall of the box was a metal response lever that, when pressed, resulted in (conditional) delivery of a standard food pellet (Noyes, $45 \mathrm{mg}$ ) into an adjacent tray from a pellet dispenser attached to the outside of the box. These response levers were $11 \mathrm{~cm}$ away from the projection panels, making it necessary for the animal to break eye contact from the visual stimulus in order to press the response lever. The requirement of a motor response spatially discontiguous from the visual stimulus was intended to emphasize further the attentional component of the task (cf. Meyer et al., 1986). Ambient illumination was from two $7.5 \mathrm{~W}$ bulbs, one above each response lever.

Behavior testing: procedure. Prior to surgery, each animal was fooddeprived to $85 \%$ of its body weight, and then shaped in the testing box to press sequentially the initiate lever, followed by one of the response 


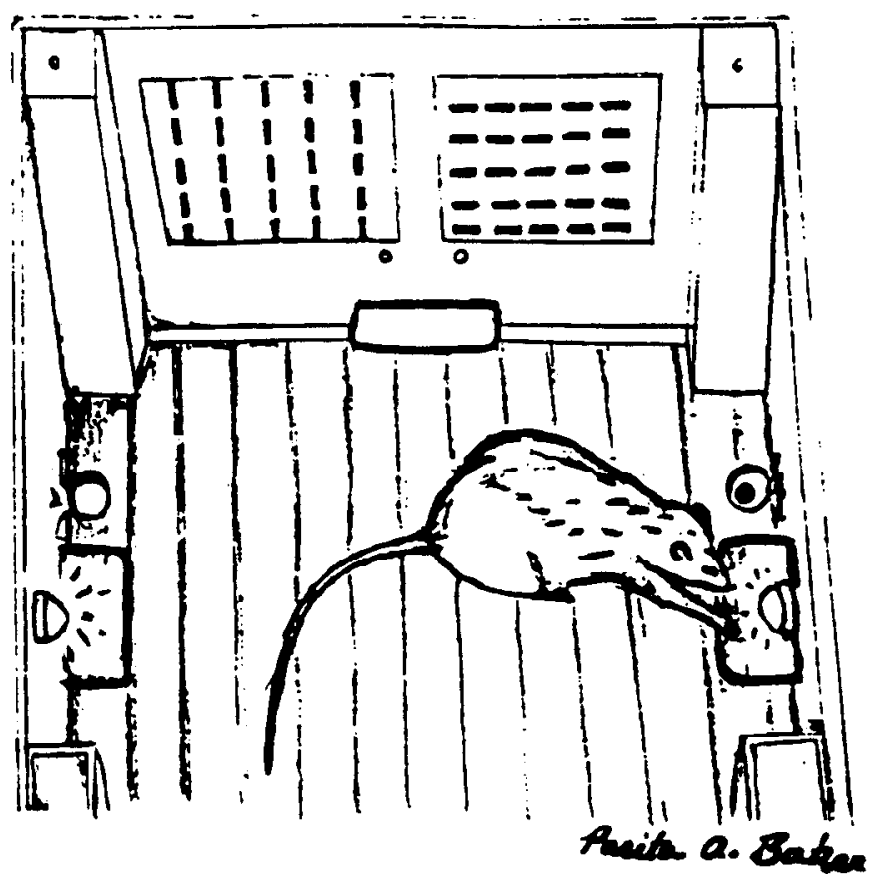

Figure 2. Visual discrimination chamber. Animal presses initiate lever between two visual display panels, then must turn and press response lever on side wall ipsilateral to correct stimulus in order to receive a food reward.

levers in order to receive a food reward. Shaping stimuli were a large circle and a small circle, differing in measured luminance by a factor of 5.5 , each projected simultaneously onto one of the two panels. Animals were shaped to press the response lever on the same side of the box as the panel on which the large circle appeared (i.e., a size/brightness visual stimulus discrimination with compatible but discontiguous response). Successful shaping required 30-40 training sessions of 30-60 min each; for this study, six rats failed to acquire the shaping task within 40 sessions and were not considered further.

Within $5 \mathrm{~d}$ of completion of shaping, the operates received lesions and osmotic pump implants. Testing began 5-7 d postoperatively. Normals began testing following a 7-10 d delay after their shaping was completed.

All animals were first tested for retention of the size/brightness discrimination, followed immediately by learning of a horizontal/vertical stripes discrimination, and then by learning of a column/row discrimination (Fig. 3). Stripes discrimination is known to be disrupted by both visual and parietal cortex lesions (McDaniel and Wall, 1988), and the more difficult column/row discrimination was thought to place an even greater demand on the animals' visual processing capabilities, and therefore be sensitive to a small lesion. The stripes were $6.5 \mathrm{~mm}$ wide, alternating black and white, with the black-white position varying randomly. Columns/rows were composed of black $6.5 \mathrm{~mm}$ squares on a white background. The patterns differed in the center-to-center ratio of the squares $(2.5: 1)$ such that they appeared as either columns or rows of squares (Dodwell, 1970; Haun et al., 1989a); the position of this pattern on the display panel shifted randomly by $6.5 \mathrm{~mm}$ either left and right (columns), or up and down (rows).
A

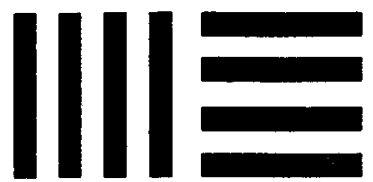

B

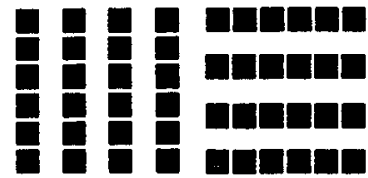

Figure 3. Stimulus pairs used in behavioral testing: $A$, Vertical versus horizontal stripes (black-white position varies randomly). $B$, Columns versus rows of squares (position of each pattern on the display panel varies; see Materials and Methods).
Each trial consisted of the animal pressing the initiate lever to produce onset of the stimulus pair, and then the correct (i.e., same-side) response lever to receive the food reward. The positive (rewarded) stimuli were the large circle, vertical stripes, and columns of squares. Pressing the correct response lever terminated the stimulus display; start of the next trial could occur at any time after the response. A press on the incorrect lever also terminated the stimulus display, and also extinguished the ambient illumination and deactivated the initiate lever, both for $30 \mathrm{sec}$. The left-right position of the correct stimulus varied according to a set pseudorandom Gellerman series (Gellerman, 1933), the starting point of which was determined randomly. A self-correction procedure was also used; that is, the same stimulus pairing was repeated until a correct response occurred. Thirty trials were given on each day, in blocks of 10 separated by 10 min during which the animal was placed in its home cage in the testing room. Testing continued until criterion or 1200 trials was reached; criterion was $90 \%$ correct responses in one 10 trial block, or $80 \%$ correct responses in two 10 trial blocks on the same day. The side of each correct and incorrect response was automatically recorded, along with the response time (i.e., the time between pressing the initiate lever and pressing a response lever).

Morphometry: lesion reconstruction. Animals receiving the UM fraction were perfused within $5 \mathrm{~d}$ of completing behavior testing (8-12 weeks postlesion), while animals receiving the CM fraction (which completed behavior testing sooner; see Results) were held until 8-9 weeks postlesion before perfusion. All animals were perfused transcardially with $4 \%$ buffered paraformaldehyde and the brains cryostat-sectioned coronally at $24 \mu \mathrm{m}$. For the operates, every eighth section through the entire neocortex was stained with cresyl violet for lesion reconstruction.

Lesion size was determined by subtraction: the volume of all gray matter in the entire neocortex was measured from planimetric sections of camera lucida drawings, separately for the operated and unoperated sides. Subtracting operated side volume from unoperated side volume yielded the volume of neocortex removed by the lesion. Additionally, lesion size was expressed as percentage neocortex removed for each animal [(unoperated side volume - operated side volume)/unoperated side volume] to account for interanimal differences in brain size and tissue shrinkage.

The extent of each lesion was also quantified, by measuring the distance from the lesion boundary to the rostral and caudal poles of the hemisphere, and to the longitudinal and rhinal sulci.

Cortical areas were identified according to the cytoarchitectonic criteria of Krieg (1946a,b), with the exception of medial frontal cortex, where the more detailed criteria of Miller and Vogt (1984) were used.

Morphometry: counts of ${ }^{3} \mathrm{H}$-thymidine-labeled neurons. Coronal sections $(24 \mu \mathrm{m})$ at $192 \mu \mathrm{m}$ intervals through the entire cortex rostral to the lesion were mounted on glass slides and dipped in Kodak NTB-2 emulsion. All slides were intermixed and coded such that subsequent cell counts were made without knowledge of either the contents of the implanted pump or the behavior results. After a constant exposure time of 3 weeks, the slides were developed and stained with cresyl violet.

Total numbers of thymidine-labeled neurons in nonmolecular layers of the medial frontal cortex of both the right (operated) and the left (unoperated) hemispheres were calculated from counts of heavily labeled cells ( $>15$ grains/nucleus), using procedures standardized in our laboratory (Cunningham et al., 1979; Haun and Cunningham, 1984, 1987; Eagleson et al., 1990, 1992). Counts are expressed as an intraanimal ratio [(operated side/unoperated side) $\times 100$ ] to account for interanimal differences in uptake and intensity of label, as well as differences in visualization of signal on sections processed at different times. The use of a relatively high grain-count criterion for a labeled cell ensures that few if any glia are included in these counts, since gliogenesis in rat cortex occurs substantially postnatally, when further cell division would dilute any prenatal labeling.

The raw counts were corrected for cell size differences (Abercrombie, 1946) after measurement of nuclear diameters; in this correction the value for section thickness was set at $2 \mu \mathrm{m}$, the upper limit of the distance a $\beta$-particle can travel in a tissue section (cf. Cunningham et al., 1979). We used the so-called "Abercrombie II" correction because Smolen et al. (1983) have shown that this procedure yields counts that accurately reflect cell number in a structure when section thickness exceeds cell size, as it does here.

Cell counts were further expressed separately for each 10 th percentile of the distance along medial frontal cortex, beginning at the caudal boundary. These data were then normalized to the caudal-rostral distance of medial frontal cortex measured in a normal control animal.

Medial frontal cortex: cytoarchitecture. Medial frontal cortex was identified by its distinct cytoarchitectonics, using criteria established in 


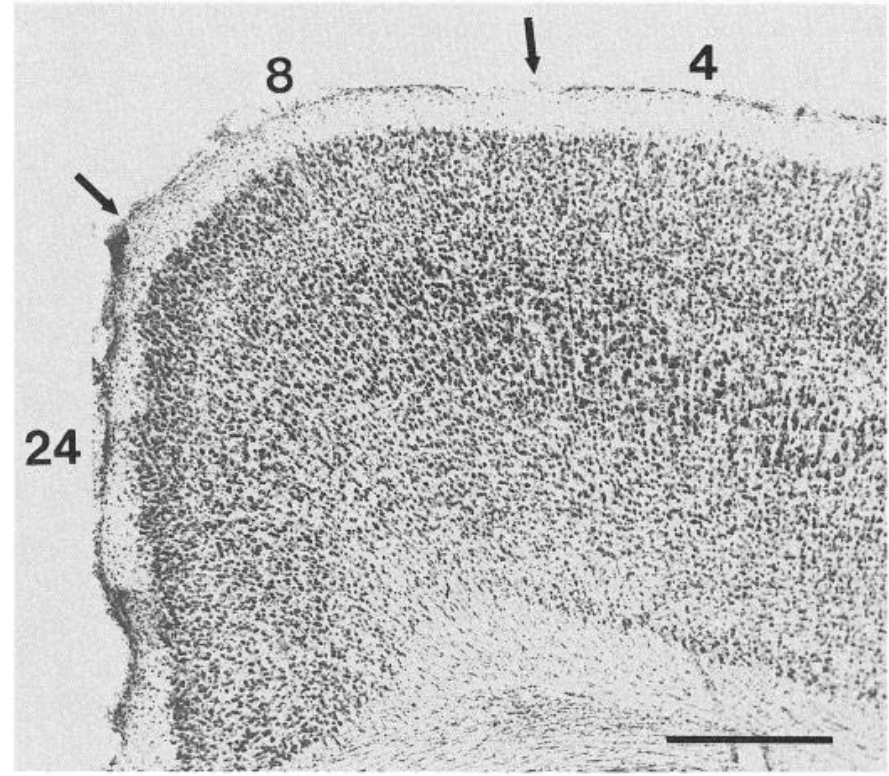

Figure 4. Coronal section through caudal area 8 of adult rat (arrows indicate boundaries). Layer IV is poorly differentiated and contains larger, lighter-staining cells, compared to area 4 laterally; cell density in layer IV is greater and layers II-III are lighter staining and poorly distinguishable, compared to area 24 ventromedially. ${ }^{3} \mathrm{H}$-thymidinelabeled cells are counted in area 8 from blind-coded sections. Cresyl violet-stained $100 \mu \mathrm{m}$ section. Scale bar, $500 \mu \mathrm{m}$.

Miller and Vogt (1984) (Fig. 4). We use their designation for this regionarea 8-which largely corresponds to PCm of Krettek and Price (1977) and, for much of its caudal-rostral extent, to Fr2 of Zilles $(1985,1990)$. This area is characterized by the absence of granular cells in layer IV, which is poorly differentiated or absent; large elliptical cell bodies in layer V; and small round somata in layer VI as well as in layers II and III, the latter two layers being poorly distinct from each other. In addition, layers V and VI comprise nearly three-fourths of its depth, notably more than in areas laterally and medioventrally. Following Miller and Vogt (1984), we also consider the caudal boundary of area 8 to be at the level of the rostral boundary of retrosplenial cortex (area 29), because the latter's unique staining pattern permits the designation of a reliable end point. Rostrally, we set the boundary of area 8 at the level of the rostral boundary of nucleus accumbens, because anterior to that point (1) there is a noticeable cytoarchitectonic change (a cell-sparse layer IV becomes distinct) and (2) few if any E19 labeled cells are seen in layers II and III in the medial aspect of the cortex.

Statistics. Comparisons of cell counts in UM and CM animals, and of error ratios and response times in all groups, were with the MannWhitney test, corrected for multiple comparisons by Dunn's procedure (Kirk, 1968). Numbers of trials to criterion were analyzed together for both the stripes and column/row tasks, using a repeated-measures ANOVA (unweighted means analysis, df reduced for missing value estimations), followed by post hoc Tukey tests for individual group comparisons. The data on lesion size were compared between UM and CM animals using $t$ tests. Correlations of cell counts and the behavior data compared linear and several polynomial regression equations (GB-STAT, $3.0)$; the model yielding the highest coefficient of determination $(\hat{\gamma})$ is reported, provided it predicts at least $10 \%$ more of the variance than the model with one less term. Mean values are expressed \pm SEM.

\section{Results}

One lesion animal that received a CM-containing pump implant developed seizures during learning of the stripes discrimination, and was not tested further; poor histology in another CM animal prevented reliable lesion reconstruction. Therefore, the behavior results reported below are from seven animals with posterior cortex lesions receiving the $\mathrm{CM}$ fraction, seven with posterior cortex lesions receiving the UM fraction, and six normal controls. Cell counts are from all the operates completing the be-

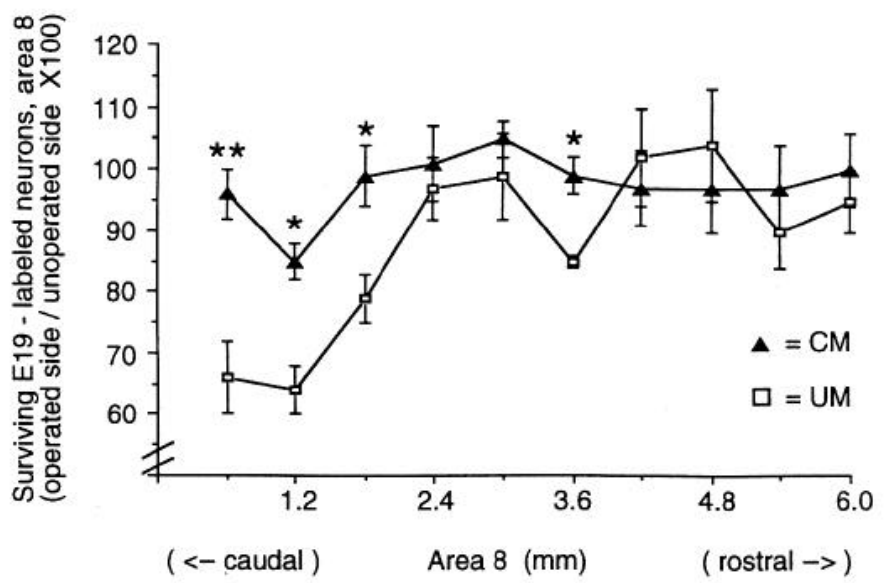

Figure 5. Remaining E19 labeled neurons in area 8 of adult rats receiving unilateral lesions in visual cortex (rostral border region) and infusions of CM or UM. Significantly more neurons remain in CMreceiving animals at $0.6 \mathrm{~mm}, 1.2 \mathrm{~mm}, 1.8 \mathrm{~mm}$, and $3.6 \mathrm{~mm}$ from the caudal boundary of area 8, compared to UM-receiving animals: $96 \pm$ $4 \%, 85 \pm 3 \%, 99 \pm 5 \%$, and $99 \pm 3 \%$ compared to $66 \pm 6 \%, 64 \pm$ $4 \%, 79 \pm 4 \%$, and $85 \pm 1 \%$, respectively. ${ }^{*}, p<0.05 ;{ }^{* *}, p<0.01$; Mann-Whitney test. $N=7$ for both CM and UM.

havior assay, and lesion data are from six of the CM and all seven of the UM animals.

\section{Lesions: reconstruction and histology}

In each animal, the lesion extended completely across the rostral borders of visual areas 18 and 17 and down to the white matter. The lesions also removed variable amounts of rostral area $18 \mathrm{a}$, lateral area 29c, and parietal areas caudal to the barrel fields of somatosensory cortex. In no animal was the lesion closer than $450 \mu \mathrm{m}$ to area 8 ; there was no significant difference between $\mathrm{CM}$ and UM animals in distance from the lesion to area 8 .

Mean lesion size in the CM animals was $7.22( \pm 1.95) \mathrm{mm}^{3}$, which is not significantly different from the $10.27( \pm 0.53) \mathrm{mm}^{3}$ in the UM animals. Expressed as percentage of neocortex removed, lesion size ranged from $7.5 \%$ to $12.3 \%$ and was not significantly different for the UM and CM groups.

Distances from the lesion to the rostral, medial, and lateral boundaries of the neocortex were not significantly different in the two groups of operates. However, distance from the lesion to the caudal pole of neocortex was greater in UM animals [3717 $( \pm 195) \mu \mathrm{m}]$ compared to CM animals [4376 $( \pm 195) \mu \mathrm{m} ; t(11)$ $=2.58 ; p<0.03]$. This measure of the caudal extent of the lesion did not correlate with neuron survival in area 8 for either group of operates, or with any of the behavioral measures.

\section{Area 8: neuron survival}

Animals receiving posterior cortex lesions plus pump implants containing the control UM fraction had significantly reduced numbers of ${ }^{3} \mathrm{H}$-thymidine-labeled cells in ipsilateral area 8, especially caudally, compared to lesion animals receiving the $\mathrm{CM}$ fraction (Fig. 5). Compared to the contralateral side, the overall reduction in numbers of labeled cells at the three caudalmost levels $(0.6 \mathrm{~mm}, 1.2 \mathrm{~mm}$, and $1.8 \mathrm{~mm}$ from the caudal boundary of area 8$)$ was $26 \%( \pm 4 \%)$ in the UM animals; the comparable reduction in $\mathrm{CM}$ animals was $8 \%( \pm 2 \%)$.

$\mathrm{CM}$ animals also had significantly more labeled cells ipsilaterally in a region $3.0-3.6 \mathrm{~mm}$ from the caudal boundary, that is, about the mid-point of area 8 . In the UM animals, the re- 

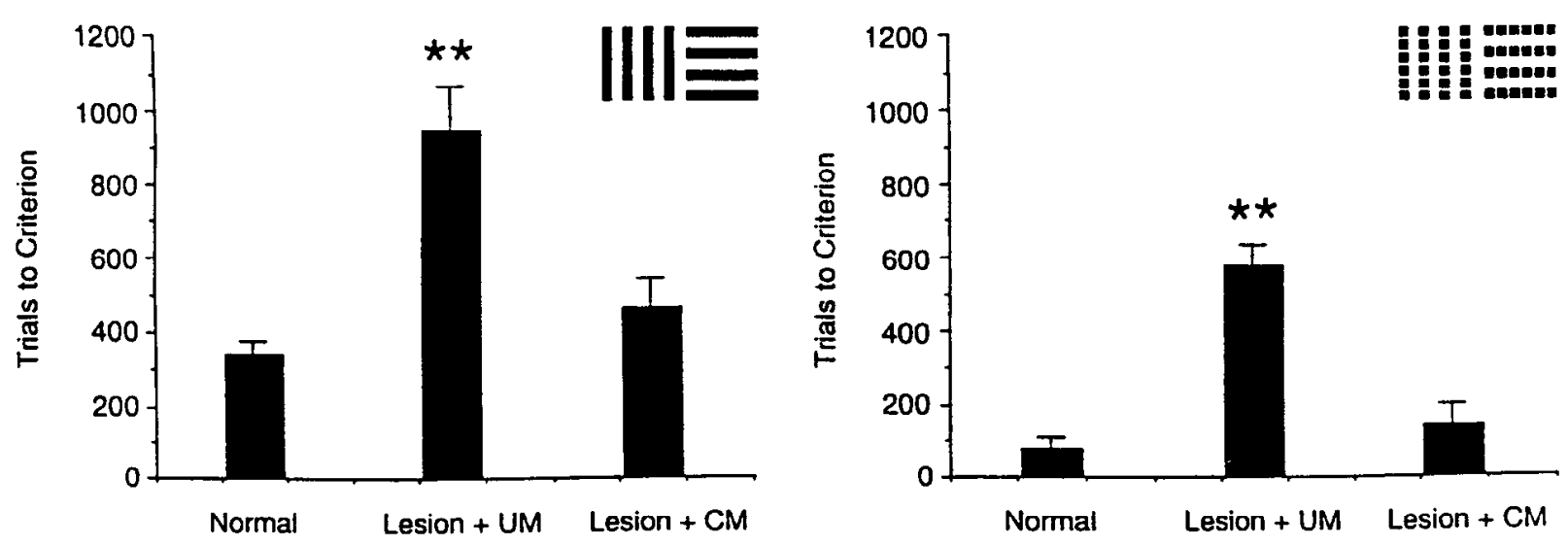

Figure 6. Number of trials to learn stripes discrimination $(A)$ followed by column/row discrimination $(B)$. Normal rats require $338( \pm 41)$ and 78 $( \pm 33)$ trials, respectively, to reach criterion. Animals receiving unilateral rostral visual cortex lesions plus infusion of UM fraction require significantly more trials, $947( \pm 120)$ and $578( \pm 58)$, respectively; animals with comparable lesions plus infusion of $\mathrm{CM}$ fraction are not significantly different from normals, requiring $459( \pm 78)$ and $141( \pm 58)$ trials, respectively. ${ }^{* *}, p<0.01$ by Tukey test. $F(2,17)=19.19 ; p<0.001$, repeated measures; ANOVA for groups. $N=7$ for CM $(A, B)$ and UM $(A), 6$ for normal $(A, B)$, and 5 for $\mathrm{UM}(B)$.

duction in number of labeled cells was small (15\% less than the number on the intact contralateral side), but it was notably consistent across animals; the ipsilateral:contralateral ratio was less than 1.0 in all UM animals, a significant difference $(p<$ 0.01 by binomial test). Morphologically, this region of area 8 is characterized by a slight lateral expansion in its supragranular layers, giving the appearance of a bump dorsally and laterally in the middle of the overall cylindrical shape of area 8.

In all animals, the labeled cells were overwhelmingly in layers II-III. Exceptions were caudally, where a few labeled cells were seen in layer $V$, and rostrally, where the molecular layer contained increasing numbers of labeled cells (not included in the counts), most of them notably smaller than in submolecular layers. There was a caudal/ventral-rostral/dorsal gradient in the labeling, with relatively morc labcled cells in the ventral portion of layers II-III caudally, and relatively more in the dorsal portion rostrally, a result identical to the patterns of rodent neurogenesis in medial frontal cortex described by Bayer and Altman (1991).

Lesion size was not significantly correlated with numbers of labeled cells in area 8 at any caudal-rostral level, for either the UM or the CM group. As for lesion extent, the only significant correlation between cell number and lesion extent was at midlevel area 8 (3.6 $\mathrm{mm}$ from the caudal boundary), where the number of labeled cells was positively correlated with distance from the lesion to the midline, at least for the UM animals ( $r_{\text {lin }}$ $=0.78 ; p<0.04)$. Thus, the farther the posterior cortex lesion extended medially in animals receiving the UM fraction, the fewer the number of labeled cells in area 8 , but only in this one region midway along the caudal-rostral extent of the area. In CM animals, where there was practically no reduction in the number of labeled cells in area 8 , the correlation with medial extent of the lesion was not significant $\left(r_{\text {lin }}=-0.12\right)$.

\section{Visual discrimination learning}

The small unilateral lesions in rostral visual/posterior parietal cortex did not impair the animals' ability to make simple visual discriminations, as indicated by their rapid relearning of the size/brightness discrimination. Animals receiving the lesion and the UM fraction relearned size/brightness in $108( \pm 57)$ trials, not significantly different from the $73( \pm 17)$ trials by normals and the $58( \pm 15)$ trials by animals receiving the lesion and the CM fraction.

Pattern discrimination learning. UM and CM animals were significantly different, however, in learning to discriminate the two pairs of visual patterns. In the horizontal/vertical stripes discrimination task, two of the seven UM animals failed to reach criterion within 1200 trials, and were assigned that number for data analyses; they were also not tested on the subsequent col$\mathrm{umn} /$ row discrimination task. All CM animals reached criterion within 1200 trials on both tasks.

UM animals required almost three times as many trials to learn the stripes discrimination, and over seven times as many trials to learn column/row discrimination, as did normals. In contrast, CM animals were not significantly different from normals in trials to criterion in either task (Fig. 6).

Errors. UM and CM animals also committed different patterns of errors. Across both tasks, UM animals made $65 \%$ more incorrect responses on the left lever (contralateral to the lesion) than incorrect responses on the right lever. This left:right error ratio of 1.65 ( 10.37 ) was significantly greater than the 0.96 $( \pm 0.07)$ left:right ratio in normals (Fig. 7). Most of the errors committed by UM animals (77\%) were perseverative, and most were contralateral to the lesion; that is, incorrect responses on the left lever that followed a left-lever response on the preceding trial (correct or incorrect) exceeded incorrect responses on the right lever that followed a right-lever response on the preceding trial (correct or incorrect) by almost 3:1. This left:right ratio of perseverative response errors in UM animals significantly exceeded the comparable ratio in normals (Fig. 8).

In contrast, $\mathrm{CM}$ animals showed no contralateral bias in their incorrect responses, either in total errors (Fig. 7) or in the subgroup of perseverative errors (Fig. 8). Indeed, all seven CM animals had an ipsilateral bias in error responses (i.e., left:right incorrect response ratios $<1$ ), a significant result by binomial test $(p<0.01)$. Furthermore, in contrast to the UM animals, this ipsilateral bias in the CM animals did not arise from perseverative errors; the mean left:right ratio of perseverative error 


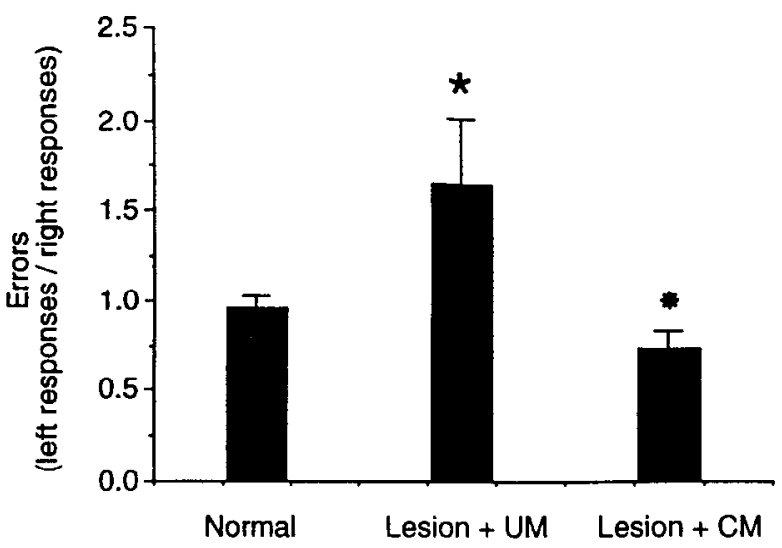

Figure 7. Incorrect responses on left lever relative to those on right lever during learning of visual discriminations. Left:right ratio is near unity for normals $[0.96( \pm 0.07)]$ and significantly greater in lesion animals receiving UM fraction [1.65 $( \pm 0.37)] .{ }^{*}, p<0.05$ by MannWhitney test. In lesion animals receiving CM fraction, ratio is significantly less than unity $[0.74( \pm 0.10) ; p<0.01$ by binomial test $] . N=7$ (CM, UM) and 6 (normal).

responses was $0.87( \pm 0.10)$, not significantly different from 1.00 .

Error response time. In both the stripes and the column/row tasks, lesion animals receiving the UM fraction were faster making incorrect responses on the left (contralateral) lever compared to making incorrect responses on the right lever. This left:right ratio in incorrect-response time $[0.61( \pm 0.10)]$ is significantly less than in normals (Fig. 9). In contrast, lesion animals receiving the $\mathrm{CM}$ fraction showed no side bias in incorrect response times. There were no significant side biases in response time for correct responses in the normal, UM, or CM groups.

\section{Discussion}

The results of this study demonstrate that (1) mature neurons in neocortex are rescued from axotomy-induced death by molecules derived from embryonic cortex tissue, (2) the rescue is chronic (at least 6 weeks after the survival-promoting factor(s) is no longer being supplied exogenously), and (3) the neuron rescue is associated with improvements in behaviors characteristic of the axotomized cortical pathway.

Target dependence by neurons in medial frontal cortex. Counts of ${ }^{3} \mathrm{H}$-thymidine-labeled cells in supragranular layers of medial frontal cortex are significantly reduced in animals that receive a medial-lateral suction lesion across the rostral border region of the right visual cortex, followed by infusion of a macromolecular fraction of UM. These labeled cells are largely if not entirely neuronal, given their morphology, laminar position, and time of labeling, as well as our strict counting procedures (see Materials and Methods). We interpret the reduction in numbers of these labeled neurons in the UM animals to result from axotomy of their projections to posterior cortical areas, in part because of known projection patterns and in part because the behavior results are what would be expected from a disconnection of the frontal-occipital pathway (see below). This finding is consistent with the idea that even mature CNS neurons may be target dependent for their survival (cf. Cunningham et al., 1987; Purves, 1990; Eagleson et al., 1992).

The neuron loss is specific to particular levels of medial frontal cortex, defined along its caudal-rostral distance. Most loss is in the caudal third of area 8 , consistent with the fact that most

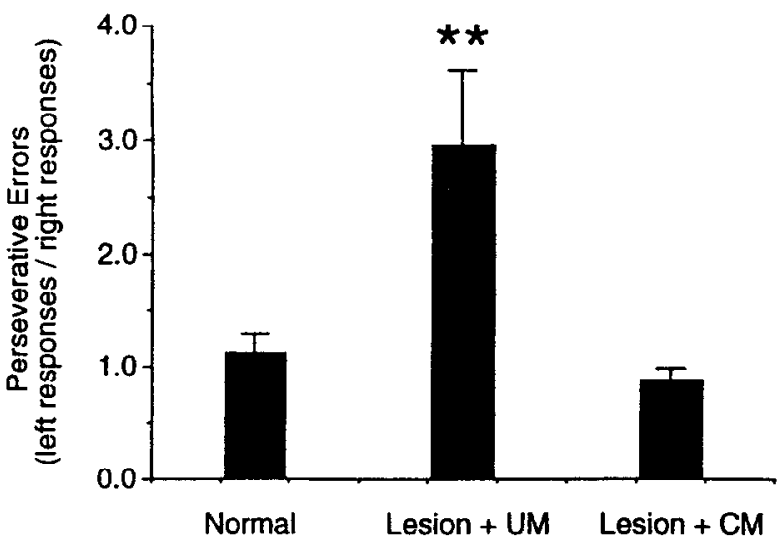

Figure 8. Perseverative incorrect responses on left lever relative to those on right lever during learning of visual discriminations. Left:right ratio is near unity for normals $[1.11( \pm 0.18)]$ and significantly greater in lesion animals receiving UM fraction $[2.96( \pm 0.67)] .{ }^{* *}, p<0.01$ by Mann-Whitney test. In lesion animals receiving $\mathrm{CM}$ fraction, ratio is not different from unity $[0.87( \pm 0.11)] . N$ valucs are as in Figure 7 .

projections from medial frontal cortex to visual cortical areas originate in caudal portions of area 8 (Miller and Vogt, 1984; Dreher et al., 1990). However, our lesions also extended into posterior parietal cortex, which receives projections from more rostral locations in area 8 (Kolb and Walkey, 1987; Reep et al., 1987). Thus, the small but consistent neuron loss at mid-level area 8 after the lesion is not surprising. The region of this loss is quite discrete, extending from about $3.0 \mathrm{~mm}$ to $3.6 \mathrm{~mm}$ from the caudal boundary, and appears to coincide with the approximate location where contralateral eye and eyelid movements are elicited by electrical stimulation most medially in frontal cortex (Hall and Lindholm, 1974) and where contralateral head movement is elicited at the lowest threshold of electrical stimulation in medial frontal cortex (Sinnamon and Galer, 1984). This particular region therefore may be an important subdivision of area 8 with respect to visuomotor control.

Neuron rescue in medial frontal cortex. At all levels of area 8 where the posterior cortex lesion results in loss of E19 labeled neurons, significantly more of these neurons are present when $\mathrm{CM}$ is infused into the lesion cavity. We interpret this result as

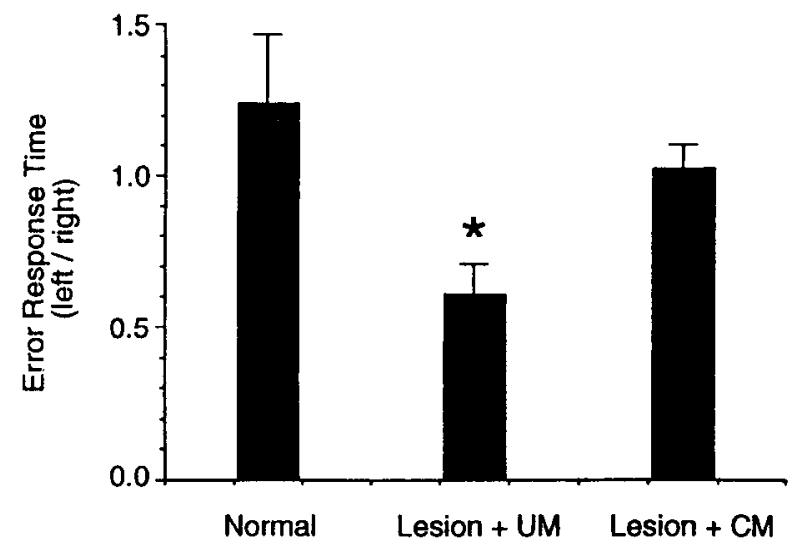

Figure 9. Time between onset of visual stimulus and incorrect responses on left lever compared to those on right lever during visual discrimination learning. Left:right ratio is $1.24( \pm 0.23)$ in normals and $1.02( \pm 0.08)$ in lesion animals receiving $C M$ fraction, but significantly less in lesion animals receiving the UM fraction $[0.61( \pm 0.10)] .{ }^{*}, p<$ 0.05 for both comparisons by Mann-Whitney test. $N=6$ for all groups. 
a rescue of neurons that otherwise die following the lesion in the UM animals. This neuron rescue appears permanent, since counts of surviving neurons were obtained 6-7 weeks after the survival-promoting factor(s) was no longer being supplied. A similar permanent rescue of neurons in area 8 is produced by embryonic cortex transplants placed in large unilateral posterior cortex lesion sites in neonates (Haun et al., 1989a).

Neurotrophic rescue of damaged adult neurons has also been reported in other CNS as well as PNS pathways. A cortically derived neuron survival factor supports long-term rescue of dLGN neurons after visual cortex lesions (Eagleson et al., 1992). A continuous application of NGF to the cut ends of axotomized mature dorsal root ganglion neurons completely prevents their death (Otto et al., 1987; Rich et al., 1987). The results of those studies, and the present study, indicate a dependence of mature neurons on neurotrophic factors for their survival.

The survival-promoting fraction of $\mathrm{CM}$ in this study is obtained from cultures of embryonic primordia from which the frontal-occipital pathway of the cortex will normally develop. However, questions about the specificity of the active factor for neurons in this pathway, or whether one or several factors are involved, cannot be answered without further purification and characterization of the active molecule(s).

Behavioral improvement in CM animals. Animals receiving small unilateral lesions in the rostral visual/posterior parietal cortex region, followed by infusion of the UM fraction, require significantly more trials to learn a horizontal versus vertical stripes discrimination, as well as a subsequent discrimination of columns versus rows of squares, compared to normals. Comparably lesioned animals receiving the CM fraction learn these discriminations in normal numbers of trials. A learning deficit in the same column/row discrimination used here also occurs following a large lesion of the posterior cortex unilaterally in neonatal rats, and is similarly spared by transplants of the same type of tissue from which the active CM fraction is derived (Haun et al., 1989a).

Plausible anatomical mechanisms underlying the behavioral recovery would include sprouting by axon collaterals of the rescued neurons, and sprouting by area 8 neurons that were not axotomized by the lesion.

Nature of the behavioral deficit. Lesions of posterior parietal cortex that include the region of our lesions have previously been shown to result in a range of deficits in visually guided behaviors (e.g., Kolb and Walkey, 1987; McDaniel and Wall, 1988). Of particular relevance to the present study is the finding that unilateral parietal lesions produce a transient deficit in orienting to visual stimuli contralaterally (Crowne et al., 1986). The deficits in visual discrimination obtained in this study resulted after smaller lesions in the parietal/occipital border region, and the deficits were chronic. This is likely due to the difficult nature of the tasks we used, especially the requirement of a response that is spatially discontiguous from the visual stimulus. In a pilot study, we found no deficits in these visual discrimination tasks following comparable lesions if a contiguous response was required (a press on the stimulus panel).

The pattern of errors committed by the animals while learning these tasks is sensitive to the posterior cortex lesion. While normals make incorrect responses equally often on the left and on the right levers, lesioned UM animals make $65 \%$ more incorrect responses on the left than on the right, and most of this difference is due to repeated left-lever responding by UM animals. This left-sided bias in perseverative errors has an es- pecially large impact on column/row learning, where the correlation between trials to criterion and left:right ratio of perseverative errors is the highest obtained in this study $\left(r_{\mathrm{log}}=\right.$ $0.92 ; p<0.04)$. The degree of contralateral bias in perseverative errors, then, is a strong predictor of how long these animals take to learn the discrimination.

In parallel with their contralateral error bias, UM animals respond faster contralaterally, but only when their responses are incorrect. We interpret these contralateral biases in both errors and incorrect response times as a reflection of the animals' failure to inhibit incorrect responses contralaterally when the correct stimulus appears ipsilaterally. An impaired inhibition of inappropriate responses contralateral to the lesion has been reported after right-hemisphere lesions in area 8 (Vargo et al., 1988), and after unilateral ibotenic acid lesions of the medial striatum (Brown and Robbins, 1989). This latter area receives direct projections from medial frontal cortex (Sesack et al., 1989), consistent with the idea that a unilateral loss of cortical control over motor response accounts for the continual incorrect responding contralaterally.

In humans, persistent incorrect or inappropriate (allesthetic) responses are a feature of frontal lobe damage (e.g., Luria, 1973; Nelson, 1976; Verfaellie and Heilman, 1987). In cases of unilateral frontal cortex damage, there is a similar tendency for these allesthetic responses to be contralateral to the lesion and to be rapid (Guitton et al., 1985; Butter et al., 1988). These behaviors have also been interpreted as failures to inhibit contralateral responding (Luria, 1973; Butter et al., 1988), and may be attributable to either loss of callosal projections, or loss of descending motor control.

Lesion animals receiving the neurotrophically active $\mathrm{CM}$ fraction, by contrast, show no contralateral bias in either error measure, or in response time, and learn both visual pattern discriminations in normal numbers of trials. Thus, the rescue of area 8 neurons in these animals by the neurotrophic fraction is associated with restored control of motor response and recovery of normal learning.

Contralateral neglect. The more common symptom of frontal cortex damage that is also seen in rats with unilateral frontal lesions is contralateral neglect of sensory stimuli (Cowey and Bozek, 1974; Barth et al., 1982; Vargo et al., 1988; King and Corwin, 1990). In the visual discrimination tasks of this study, a contralateral sensory neglect would result in an ipsilateral bias in response errors, that is, neglect of, or inattention to, contralateral visual stimuli that are correct leads to more ipsilateral responses that are incorrect.

The only group in this study showing an ipsilateral error bias is the CM animals, all of whom have left:right incorrect response ratios of $<1$ (relatively more incorrect responses ipsilateral to the lesion). This ipsilateral bias is not due to perseveration, that is, the bias does not reflect a persisting failure to inhibit incorrect responding, in contrast to the contralateral error bias in the UM animals.

An ipsilateral error bias would not be surprising in our operates, given that our direct lesions remove neurons in posterior parietal cortex; as indicated above, unilateral parietal lesions have been shown to produce contralateral sensory neglect (Crowne et al., 1986). However, since our lesions are small, sensory neglect may be a minor component of the behavioral deficits, especially with our testing procedure, which places a strong demand on attentional control of the motor response (Meyer et al., 1986). In the CM animals, this motor control is 
restored, and the sensory neglect is then seen in the ipsilateral error bias.

Thus, the results of this study suggest that recovery of particular functions by neurotrophic rescue of particular neuron populations in adults is feasible. Furthermore, this sparing of a neuronal population that subserves a particular component of performance may unmask other deficits subserved by damaged neuronal populations not rescued by the neurotrophic factor.

\section{References}

Ahercrombie M (1946) Estimation of nuclear population from microtome section. Anat Rec 94:238-248.

Aldskogius H, Arvidsson J (1978) Nerve cell degeneration and death in the trigeminal ganglion of the adult rat following peripheral nerve transection. J Neurocytol 7:229-250.

Barth TM, Parker SM, Sinnamon HM (1982) Unilateral lesions of the anteromedial cortex in the rat impair approach to contralateral visual cues. Physiol Behav 25:141-147.

Bayer SA, Altman J (1991) Neocortical development. New York: Kaven.

Bleier R (1969) Retrograde transsynaptic cellular degeneration in mammillary and ventral tegmental nuclei following limbic decortication in rabbits of various ages. Brain Res 15:365-393.

Brown VJ, Robbins TW (1989) Elementary processes of response selection mediated by distinct regions of the striatum. J Neurosci 9:3760-3765.

Butter CM, Rapcsak S, Watson RT, Heilman KM (1988) Changes in sensory inattention, directional motor neglect and "release" of the fixation reflex following a unilateral frontal lesion: a case study. Neuropsychologia 26:533-545.

Cowey A, Bozek T (1974) Contralateral "neglect" after unilateral dorsomedial prefrontal lesions in rats. Brain Res 72:53-63.

Crowne DP, Richardson CM, Dawson KA (1986) Parietal and frontal eye field neglect in the rat. Behav Brain Res 22:227-231.

Cunningham TJ, Huddleston C, Murray M (1979) Modification of neuron numbers in the visual system of the rat. J Comp Neurol 184: 423-434.

Cunningham TJ, Sutilla CB, Haun F (1987) Trophic effects of transplants following damage to the cerebral cortex. Ann NY Acad Sci 495:153-168.

Cunningham TJ, Fisher C, Haun F (1988) Testing the trophic factor hypothesis in the visual system. Neurol Neurobiol 48:89-104.

Dodwell PC (1970) Anomalous transfer effects after pattern discrimination training in rats and squirrels. J Comp Physiol Psychol 71:4251.

Dreher B, Dehay C, Bullier J (1990) Bihemispheric collateralization of the cortical and subcortical afferents to the rat's visual cortex. Eur J Neurosci 2:317-331.

Eagleson KL, Haun F, Cunningham TJ (1990) Different populations of dorsal lateral geniculate nucleus neurons have concentration-specific requirements for a cortically derived neuron survival factor. Exp Neurol 110:284-290.

Eagleson KL, Cunningham TJ, Haun F (1992) Rescue of both rapidly and slowly degenerating neurons in the dorsal lateral geniculate nucleus of adult rats by a cortically derived neuron survival factor. Exp Neurol 116:156-162.

Gage FH, Armstrong DM, Williams LR, Varon S (1988) Morphological response of axotomized septal neurons to nerve growth factor. J Comp Neurol 269:147-155.

Gellerman LW (1933) Chance orders of alternating stimuli in visual discrimination experiments. J Genet Psychol 42:206-208.

Guitton D, Buchtel HA, Douglas RM (1985) Frontal lobe lesions in man cause difficulties in suppressing reflexive glances and in generating goal-directed saccades. Exp Brain Res 58:455-472.

Hall RD, Lindholm EP (1974) Organization of motor and somatosensory neocortex in the albino rat. Brain Res 66:23-38.

Haun F, Cunningham TI (1984) Cortical transplants reveal CNS trophic interactions in situ. Dev Brain Res 15:290-294.

Haun F, Cunningham TJ (1987) Specific neurotrophic interactions between cortical and subcortical visual structures in developing rat: in vivo studies. J Comp Neurol 256:561-569.

Haun F, Cunningham TJ, Rothblat LA (1989a) Neurotrophic and behavioral effects of occipital cortex transplants in newborn rats. Vis Neurosci 2:189-198.

Haun F, Perry R, Cunningham TJ (1989b) Long-term rescue of damaged frontal cortex neurons and sparing of visually guided behavior by acute administration of cortex conditioned medium fraction. Soc Neurosci Abstr 15:3.

Hefti $F$ (1986) Nerve growth factor promotes survival of scptal cholinergic neurons after fimbrial transections. J Neurosci 6:2155-2162.

Hefti F, Hartikka J, Knusel B (1989) Function of neurotrophic factors in the adult and aging brain and their possible use in the treatment of neuro-degenerative diseases. Neurobiol Aging 10:515-533.

Himes BT, Tessler A (1989) Death of some dorsal root ganglion neurons and plasticity of others following sciatic nerve section in adult and neonatal rats. J Comp Neurol 284:215-230.

Himes BT, Goldberger ME, Tessler A (1990) Grafts of fetal CNS tissue rescue axotomized Clarke's nucleus neurons in adult and neonatal operates. Soc Neurosci Abstr 16:818.

King V, Corwin JV (1990) Neglect following unilateral ablation of the caudal but not the rostral portion of medial agranular cortex of the rat and the therapeutic effect of apomorphine. Behav Brain Res 37: $169-184$.

Kirk RE (1968) Experimental design: procedures for the behavioral sciences. Belmont, CA: Brooks/Cole.

Kolb B, Walkey J (1987) Behavioral and anatomical studies of the posterior parietal cortex in the rat. Behav Brain Res 23:127-145.

Krettek JE, Price JL (1977) The cortical projections of the medial dorsal nucleus and adjacent thalamic nuclei in the rat. J Comp Neurol $171: 157-192$

Krieg WJS (1946a) Connections of the cerebral cortex. I. The albino rat. A. Topography of the cortical areas. J Comp Neurol 84:221-276.

Krieg WJS (1946b) Connections of the cerebral cortex. I. The albino rat. B. Structure of the cortical areas. J Comp Neurol 84:277-284.

Kromer LF (1987) Nerve growth factor treatment after brain injury prevents neuronal death. Science 235:214-216.

Lashley KS (1941) Thalamo-cortical connections of the rat's brain. J Comp Neurol 75:67-121.

Luria AR (1973) The working brain (B Hugh, trans). New York: Basic. McDaniel WF, Wall TT (1988) Visuospatial functions in the rat following injuries to striate, peristriate, and parietal neocortical sites. Psychobiology 16:251-260.

Meyer PM, Meyer DR, Cloud MD (1986) Temporal neocortical injuries in rats impair attending but not complex visual processing. Behav Neurosci 100:845-851.

Miller MW, Vogt BA (1984) Direct connections of rat visual cortex with sensory, motor, and association cortices. J Comp Neurol 226: 184-202.

Montero CN, Hefti F (1988) Rescue of lesioned septal cholinergic neurons by nerve growth factor: specificity and requirement for chronic treatment. J Neurosci 8:2986-2999.

Nelson HE (1976) A modified card sorting test sensitive to frontal lobe defects. Cortex 12:313-324.

Otto D, Unsicker K, Grothe C (1987) Pharmacological effects of nerve growth factor applied to the transectioned sciatic nerve on neuron death in adult rat dorsal root ganglia. Neurosci Lett 83:156-160.

Purves D (1975) Functional and structural changes in mammalian neurons following interruption of their axons. J Physiol (Lond) 252: 429-463.

Purves D (1988) Body and brain; a trophic theory of neural connections. Cambridge, MA: Harvard UP.

Reep RL, Corwin JV, Hashimoto A, Watson RT (1987) Efferent connections of the rostral portion of medial agranular cortex in rats. Brain Res Bull 19:203-221.

Rich KM, Luszczynski JR, Osborne PA, Johnson EM Jr (1987) Nerve growth factor protects adult sensory neurons from cell death and atrophy caused by nerve injury. J Neurocytol 16:261-268.

Rosenberg MB, Friedmann T, Robertson RC, Tuszynski M, Wolff JA Breakefield XO, Gage FH (1988) Grafting genetically modified cells to the damaged brain: restorative effects of NGF expression. Science 242:1575-1578.

Sesack SR, Deutch AY, Roth RH, Bunney BS (1989) Topographical organization of the efferent projections of the medial prefrontal cortex in the rat: an anterograde tract-tracing study with Phaseolus vulgaris leuco-agglutinin. J Comp Neurol 290:213-242.

Sinnamon HM, Galer BS (1984) Head movements elicited by electrical stimulation of the anteromedial cortex of the rat. Physiol Behav 33:185-190. 
Smolen AJ, Wright LL, Cunningham TJ (1983) Neuron numbers in the superior cervical ganglion of the rat: a critical comparison of methods for cell counting. J Neurocytol 12:739-750.

Thoenen H (1991) The changing scene of neurotrophic factors. Trends Neurosci 14:165-170.

Vargo JM, Corwin JV, King V, Reep RL (1988) Hemispheric asymmetry in neglect produccd by unilateral lesions of dorsomedial prefrontal cortex in rats. Exp Neurol 102:199-209.

Varon S, Hagg T, Manthorpe M (1989) Neuronal growth factors. In: Frontiers in clinical neuroscience, Vol 6, Neural regeneration and transplantation (Seil FJ, ed), pp 101-121. New York: Liss.
Verfaellie M, Heilman KM (1987) Response preparation and response inhibition after lesions of the medial frontal lobe. Arch Neurol 44: 1265-1271.

Will B, Hefti F (1985) Behavioural and neurochemical effects of chronic intraventricular injections of nerve growth factor in adult rats with fimbria lesions. Behav Brain Res 17:17-24.

Zilles K (1985) The cortex of the rat: a stereotaxic atlas. Berlin: Springer.

Zilles K (1990) Anatomy of the neocortex: cytoarchitecture and myeloarchitecture. In: The cerebral cortex of the rat (Kolb B, Tees RC, eds), pp 77-112. Cambridge, MA: MIT. 